\title{
Ce que vous devez savoir pour votre formation postgraduée
}

\author{
Christoph Hänggeli \\ Avocat, MPA unibe, directeur de I'Institut suisse pour la formation médicale postgraduée et continue (ISFM)
}

\section{A quelles exigences faut-il satisfaire pour obtenir un titre de spécialiste?}

La Réglementation pour la formation postgraduée (RFP) décrit les principes généraux, et communs à toutes les disciplines, de la formation postgraduée des médecins. A cela s'ajoute un programme de formation postgraduée pour chaque titre de spécialiste, qui énonce les exigences spécifiques pour obtenir le titre. Prenez le temps de les lire attentivement pour vous familiariser avec votre cursus et informez-vous des éventuelles révisions. Toutes les conditions importantes sont énoncées au chiffre 2 du programme de votre discipline (p. ex. catégories des années de formation à accomplir, cours à suivre, publications, etc.). Par ailleurs, le registre de l'ISFM vous indique tous les établissements de formation postgraduée reconnus et habilités à enseigner la formation dans leur spécialisation respective. IMPORTANT: toute activité effectuée dans un hôpital, une clinique ou un cabinet médical dont la reconnaissance ne figure pas dans le registre ne sera pas validée! Exigez un contrat écrit à chaque poste de formation postgraduée.

www.siwf.ch $\rightarrow$ Domaines spécialisés $\rightarrow$ Titres de spécialiste et formations approfondies (formation postgraduée) www.registre-isfm.ch

www.siwf.ch $\rightarrow$ Formation postgraduée $\rightarrow$ Pour les responsables des établissements de formation postgraduée $\rightarrow$ Modèle de contrat de formation postgraduée

\section{Comment documenter la formation postgraduée?}

Le logbook électronique personnel permet de consigner régulièrement toutes les exigences de formation accomplies. Il comporte entre autres le certificat ISFM avec lequel le responsable de l'établissement reconnu atteste la période de formation, les entretiens d'évaluation ainsi que les prestations et les progrès accomplis. Au terme de votre formation postgraduée, vous déposez votre demande de titre avec le logbook électronique complet et dûment rempli. Enregistrez-vous dès le début de votre formation pour le logbook électronique et remplissez-le régulièrement.

\section{La formation postgraduée peut-elle être accomplie à temps partiel?}

Oui, mais à certaines conditions. Seuls les postes à un taux d'activité d'au moins 50\% peuvent être validés. Si le programme de formation ne spécifie aucune disposition particulière, c'est la règle suivante qui s'applique: la moitié au plus de la formation postgraduée spécifique peut être accomplie à temps partiel. En revanche, la formation postgraduée non spécifique peut être entièrement accomplie à temps partiel. La plupart des programmes de formation postgraduée spécifient que la totalité de la formation peut être accomplie à temps partiel.

www.siwf.ch $\rightarrow$ Formation postgraduée $\rightarrow$ Bases réglemen taires de la formation postgraduée $\rightarrow$ Réglementation pour la formation postgraduée (RFP); art. 32

\section{Peut-on accomplir la formation postgraduée à l'étranger?}

Avant d'accepter un poste à l'étranger, sollicitez au préalable l'accord de la Commission des titres pour être certain que votre formation pourra être validée sans problème. En principe, toute formation suivie à l'étranger auprès d'un établissement de formation équivalent est reconnue. Mais en règle générale, au moins deux ans de la formation spécifique doivent être accomplis dans un établissement de formation reconnu en Suisse. Tenez également compte des dispositions particulières de votre programme en ce qui concerne la définition de la formation postgraduée spécifique, la classification des établissements de formation et la validation des opérations. Pour les titres de spécialiste ainsi que pour la plupart des formations approfondies, la formation postgraduée peut être entièrement suivie à l'étranger. Utilisez toujours le logbook électronique pour déposer vos demandes.

www.siwf.ch $\rightarrow$ Formation postgraduée $\rightarrow$ Bases réglemen taires de la formation postgraduée $\rightarrow$ Réglementation pour la formation postgraduée (RFP); art. 33 (et son interprétation) www.siwf.ch $\rightarrow$ Thèmes $\rightarrow$ Relations internationales $\rightarrow$ Formation postgraduée et activité à l'étranger 


\section{Existe-t-il une durée minimale pour la validation d'une période de formation postgraduée?}

Oui. Seules sont reconnues les périodes de formation postgraduée de six mois au moins accomplies auprès d'un même établissement de formation (en cas de formation à temps partiel: la durée est prolongée au prorata). Au maximum trois périodes courtes d'au moins trois mois sont admises par titre de spécialiste (pour chaque formation approfondie, seule une période courte peut être validée). L'assistanat au cabinet peut être validé à partir d'une période ininterrompue d'un mois déjà et ne compte pas comme une période courte.

www.siwf.ch $\rightarrow$ Formation postgraduée $\rightarrow$ Bases réglementaires de la formation postgraduée $\rightarrow$ Réglementation pour la formation postgraduée (RFP); art. 30

\section{La grossesse/maternité peut-elle être validée comme formation postgraduée?}

Si les absences mentionnées dans un certificat ISFM sont liées à une grossesse/maternité, vous n'avez pas à les rattraper pour autant que le cumul des absences par discipline et par année n'excède pas huit semaines. Si vous n'avez pas atteint la limite maximale d'absences admise, vous pouvez également demander la validation de la période de grossesse/maternité indépendamment d'un poste de formation. La réglementation précise se trouve à l'art. 31 de la Réglementation pour la formation postgraduée (RFP).

www.siwf.ch $\rightarrow$ Formation postgraduée $\rightarrow$ Bases réglementaires de la formation postgraduée $\rightarrow$ Réglementation pour la formation postgraduée (RFP); art. 31 (et son interprétation)

\section{L'assistanat au cabinet médical ou une activité de remplaçant sont-ils validés?}

L'assistanat en cabinet et le remplacement sont validés dans le cadre défini par le programme de formation postgraduée. Pour le reste, c'est l'art. 34 de la Réglementation pour la formation postgraduée qui s'applique.

www.siwf.ch $\rightarrow$ Formation postgraduée $\rightarrow$ Bases réglemen taires de la formation postgraduée $\rightarrow$ Réglementation pour la formation postgraduée (RFP); art. 34

\section{Une période de formation postgraduée peut-elle être validée simultanément pour deux titres de spécialiste différents?}

Oui, c'est possible. Une année de médecine interne générale en catégorie A peut par exemple être simultanément validée pour les titres de spécialiste en méde- cine interne générale, cardiologie, pneumologie ainsi que psychiatrie et psychothérapie. Cela facilite ainsi l'obtention de plusieurs titres de spécialiste. En revanche, il est exclu de cumuler deux activités à plein temps dans deux spécialisations (on ne peut pas accomplir un an de médecine interne générale et un an de médecine intensive dans la même année).

www.siwf.ch $\rightarrow$ Formation postgraduée $\rightarrow$ Bases réglementaires de la formation postgraduée $\rightarrow$ Réglementation pour la formation postgraduée (RFP); art. 29

\section{Faut-il réussir l'examen de spécialiste?}

Oui. Toutefois, avant de pouvoir obtenir le titre de spécialiste, vous devez également répondre à toutes les autres conditions mentionnées dans le programme de formation. Seuls les détenteurs d'un diplôme fédéral de médecin ou d'une reconnaissance de leur diplôme de médecin de l'UE sont autorisés à passer l'examen de spécialiste. Les dates et les modalités d'inscription sont à chaque fois publiées six mois avant la date de l'examen sur le site de l'ISFM.

L'organisation et le déroulement de l'examen sont du ressort des sociétés de discipline médicale.

En cas d'échec, le candidat a le droit de consulter sa copie et il a la possibilité de faire opposition. Veuillez lire la notice à ce sujet.

www.siwf.ch $\rightarrow$ Domaines spécialisés $\rightarrow$ Titres de spécialiste et formations approfondies (Formation postgraduée) $\rightarrow$ p. ex. Médecine interne générale $\rightarrow$ Examen de spécialiste www.fmh.ch $\rightarrow$ De la FMH $\rightarrow$ Organisations de médecins $\rightarrow$ Sociétés de discipline

www.siwf.ch $\rightarrow$ Domaines spécialisés $\rightarrow$ Titres de spécialiste et formations approfondies (Formation postgraduée) $\rightarrow$ p. ex Médecine interne générale $\rightarrow$ Examen de spécialiste $\rightarrow$ Informations importantes

\section{Comment déposer une demande de titre?}

La demande d'octroi d'un titre de spécialiste ou de formation approfondie peut être directement déposée via le logbook électronique. Au moyen de la liste de contrôle, assurez-vous d'avoir réuni l'ensemble des documents mentionnés dans le programme de formation postgraduée. Veuillez à ne remettre que des photocopies bien lisibles (pas d'originaux!) de tous les documents requis. Le secrétariat de l'ISFM retournera les dossiers incomplets! Seuls les certificats ISFM munis de votre signature et de celle du responsable autorisé de l'établissement de formation reconnu peuvent être validés.

www.siwf.ch $\rightarrow$ Formation postgraduée $\rightarrow$ e-logbook 


\section{Faut-il envoyer chaque année un certificat ISFM?}

Non. N'envoyez aucun certificat avant d'avoir rassemblé l'ensemble des justificatifs demandés par le programme de formation. Mais n'oubliez pas que toutes les périodes de formation postgraduée doivent être sanctionnées par un certificat ISFM valide dans lequel est consigné le résultat de l'entretien d'évaluation mené au moins une fois par an par le responsable de la formation postgraduée.

www.siwf.ch $\rightarrow$ Formation postgraduée $\rightarrow$ e-logbook

\section{Quand un programme de formation postgraduée est-il révisé?}

Les exigences spécifiques aux différents programmes de formation postgraduée évoluent car la médecine est en constante évolution. De ce fait, vérifiez régulièrement si le cursus que vous avez choisi a été actualisé ou non. En règle générale, une période transitoire de trois à cinq ans encadre l'introduction de nouvelles exigences. En d'autres termes, le candidat qui achève sa formation postgraduée dans un délai de trois à cinq ans à partir de la mise en vigueur d'un nouveau programme peut l'accomplir selon les dispositions de l'ancien programme. Au-delà de ce délai, il devra remplir les exigences du nouveau programme.

www.siwf.ch $\rightarrow$ Domaines spécialisés $\rightarrow$ Titres de spécialiste et formations approfondies (Formation postgraduée)

\section{Faut-il être membre de la FMH pour obtenir un titre de spécialiste, une forma- tion approfondie ou une attestation de formation complémentaire?}

Non. Si vous souhaitez obtenir un titre de spécialiste, une formation approfondie ou une attestation de formation complémentaire, l'affiliation à la FMH ou à la société de discipline concernée est facultative. Veuillez vous renseigner sur les avantages liés à une telle affiliation.

www.fmh.ch $\rightarrow$ Services $\rightarrow$ Pour les membres $\rightarrow$ Adhésion

\section{De quels émoluments faut-il s'acquitter?}

Veuillez vous référer au tarif des émoluments sur notre site internet. L'octroi d'un titre de spécialiste coûte CHF 4000. Un montant maximal de CHF 1000 est remboursé sur la cotisation à la FMH après acquisition du premier titre de spécialiste (CHF 200 par année d'affiliation pendant cinq ans au plus). Tout titre de spécialiste supplémentaire coûte CHF 1000, toute formation approfondie CHF 500.

www.siwf.ch $\rightarrow$ Formation postgraduée $\rightarrow$ Tarifs

\section{Peut-on évaluer un établissement de formation postgraduée?}

L'ISFM réalise chaque année une enquête auprès de tous les médecins-assistants dans le but d'établir une évaluation détaillée des établissements de formation. En participant activement à cette enquête, vous contribuez à ce que l'ISFM améliore la formation postgraduée des médecins.

www.siwf.ch $\rightarrow$ Formation postgraduée $\rightarrow$ Généralités $\rightarrow$ Enquête sur la qualité de la formation postgraduée

\section{Qui répond aux questions en matière de formation postgraduée?}

Le secrétariat de l'Institut suisse pour la formation médicale postgraduée et continue (ISFM) est à votre entière disposition pour répondre à toutes les questions relevant de la formation des médecins. Sur notre site internet, vous trouverez toutes les informations importantes. Mais nous répondons aussi personnellement à toutes vos questions (tél. 03135911 11) pendant les heures de bureau (lu-ve de $8 \mathrm{~h}$ à $12 \mathrm{~h}$ et de $13 \mathrm{~h}$ à $17 \mathrm{~h}$ ). Vous aurez sans doute plus de chance de nous atteindre par courrier électronique (siwf[at]fmh.ch).

www.siwf.ch $\rightarrow$ Domaines spécialisés $\rightarrow$ Titres de spécialiste et formations approfondies (Formation postgraduée) 